\title{
The influence of bite force strength on brain activity: A functional magnetic resonance imaging study
}

\author{
Takeo Kanayama*, H. Miyamoto, A. Yokoyama, T. Takahashi, Y. Shibuya \\ Dept. of Oral and Maxillofacial Surgery, Nagoya City University Graduate School of Medical Sciences, Nagoya, Japan
}

Received: November 28, 2014

DOI: $10.5430 /$ jbgc.v5n1p28
Accepted: December 29, 2014 Online Published: January 19, 2015

URL: http://dx.doi.org/10.5430/jbgc.v5n1p28

\begin{abstract}
In recent years, functional magnetic resonance imaging has been used to determine the interaction between chewing and brain activity. However, the factors influencing the activity of the motor cortex have not been fully elucidated. Therefore, the present study investigated the influence of the magnitude of bite force on brain activity. Fifteen right-handed healthy subjects (24-32 years; mean age, 27.8 years) were included. Sustained, constant clenching with small and large forces comprised the motor task. The spatial extent of the functional magnetic resonance imaging signal in the primary sensorimotor cortex increased with an increasing bite force in all subjects. These findings indicated the possibility of measuring the activated area in the primary sensorimotor cortex during clenching using functional magnetic resonance imaging, which revealed that the brain activity was related to the magnitude of the bite force.
\end{abstract}

Key Words: Functional magnetic resonance imaging, Bite force, Brain activity, Motor cortex, Masticatory muscles, Clenching

\section{Introduction}

In recent years, functional magnetic resonance imaging (fMRI) has been employed to delineate the interaction between chewing and brain activity. ${ }^{[1-4]}$ Brain activation associated with gum chewing results in an increase in fMRI signals; however, the factors that influence motor cortex activity have not been fully elucidated. Chewing is a complicated movement that involves opening and closing of the mouth and includes several parameters, such as the force, direction, and speed of chewing. Therefore, simple tasks are useful for investigating the manner in which each parameter influences brain activities.

Animal studies have demonstrated that several cortical and subcortical motor areas include cells with discharge rates that correlate with the parameters of the specific motor task, including the force, direction, and frequency of movement. ${ }^{[5-8]}$ Several human studies on brain mapping have focused on the relationship between brain activation and force, ${ }^{[9-13]}$ and the majority employed the distal upper extremity. Furthermore, increased force has been associated with regionally increased activation in the contralateral primary motor cortex in some, ${ }^{[9-11]}$ but not other studies. ${ }^{[12,13]}$ Each of these studies involved a different motor task for brain activation, and this could be the reason for the varying conclusions. Therefore, the present study used a clenching task to investigate the influence of bite force strength on brain activity. The clenching task is a unique task because it involves the simultaneous recruitment of the bilateral masticatory muscles, enabling the measurement of activated areas in both sensorimotor cortices and their comparison.

\footnotetext{
*Correspondence: Takeo Kanayama; Email: tkokanayama1@mac.com; Address: Dept. of Oral and Maxillofacial Surgery, Nagoya City University Graduate School of Medical Sciences, 1-Kawasumi, Mizuho-cho, Mizuho-ku, Nagoya, Japan.
} 


\section{Materials and methods}

\subsection{Subjects}

Fifteen right-handed healthy subjects (24-32 years; mean age, 27.8 years) participated in this study, which was approved by the ethics committee of the Hokkaido University Graduate School of Dental Medicine. Written informed consent was obtained from all subjects before study initiation.

\subsection{Motor task}

Sustained, constant clenching with small and large forces was used as the specific motor task in this study. Each subject individually defined small and large forces. Before scanning, each subject underwent a brief training program for the motor task and was asked to clench a dental prescale film (Fuji film) using the two magnitudes of force. Consequently, each subject could demonstrate consistent and repeated clenching with two difference magnitudes of force.

\subsection{Functional MRI}

fMRI was performed using a 1.5-T Sigma Horizon LX (GE Medical Systems, Milwaukee, WI) with a standard head coil, $\times 200-\mathrm{mm}$ field of view, and $\times 64$ matrix $\left(9.77-\mathrm{mm}^{2}\right.$ pixel size) and a blood oxygen level-dependent (BOLD) gradient-recalled echo-planar sequence (repetition time, 3,000 ms; echo time, $60 \mathrm{~ms}$; flip angle, $90^{\circ}$ ). The subject lay in the supine position in the magnet, with the head fixed by a bitemporal clamp.
The imaging protocol is shown in Figure 1. Three sagittal slices were obtained to identify the anterior and posterior commissures (AC-PC). Five 5-mm-thick slices parallel to the AC-PC line and separated by $1.5 \mathrm{~mm}$ were selected. The scanner was set in the acquisition mode for $15 \mathrm{~s}$ before each session so that steady-state transverse magnetization was achieved. Five images per slice were collected during a 25 -s rest period and again during $25 \mathrm{~s}$ of the task. This was repeated five times, providing a total of $50 \mathrm{im}-$ ages per slice. Each individual task using small and large clenching forces was separately imaged. At study termination, anatomical images were obtained using a T1-weighted spin-echo sequence to provide detailed anatomical information. Data were discarded from acquisitions in which the subject's head moved greater than $0.5 \mathrm{~mm}$, as determined using free software to calculate the head motion (SPM96; Institute of Neurology; London, UK). Acquisitions were repeated in three subjects on separate days to confirm the data reproducibility and reliability.

\subsection{Data analysis}

For each fMRI session, data were analyzed using the cross correlation (sine) method using MR Vision. The time course of the BOLD response profiles was correlated with the on/off cycles of the task. Activated pixels were generated by statistical analysis using a two-tailed t-test for comparing the active and resting conditions of the task (on/off) synchronized with the estimated sine curve. A $p$-value of $<$ .000001 was considered statistically significant.

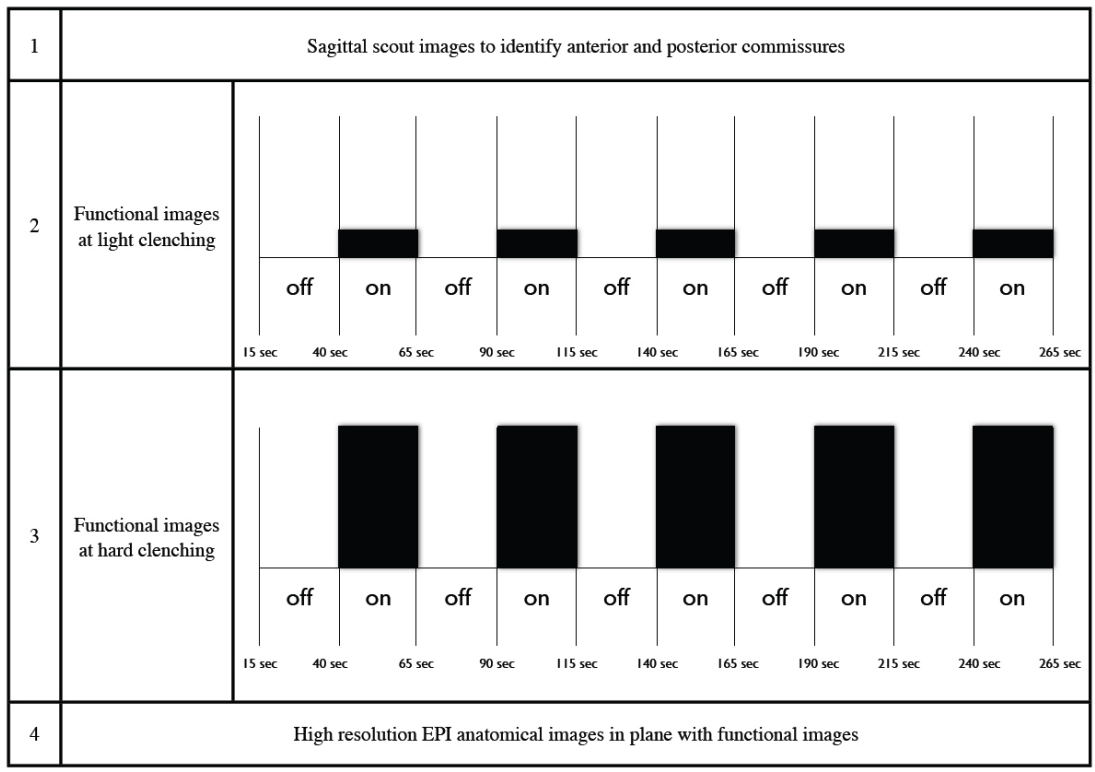

Figure 1: Functional magnetic resonance imaging protocol used in this study
The pixels were compared with the T1-weighted MR images for anatomical observations, and changes in the signal intensity in each pixel were individually examined. The number of significantly activated pixels was counted within both the left and right sensorimotor cortices. Because clenching recruits the bilateral muscles, comparisons were made between the right and left hemispheres during the clenching task performed with small and large forces using the Wilcoxon test. 


\section{Results}

Figure 2 shows an example of fMRI findings for a typical subject during the clenching task with small (yellow pixels) and large (yellow and red pixels) bite forces. We separately counted the number of activated pixels in the right and left hemispheres. The number of activated pixels at each magnitude of force is shown in the table.

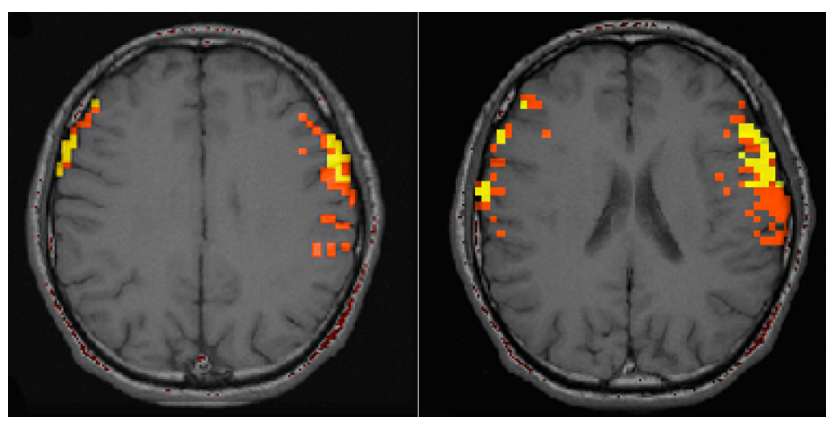

Figure 2: Functional magnetic resonance imaging (fMRI) responses for two consecutive slices covering the region of activation in the central sulcus with small (yellow pixels) and large (yellow plus red pixels) clenching forces in a typical subject. The extent of the fMRI signal increases with increasing force within and across slices.

\subsection{Right hemisphere}

During clenching with a small force, a small number of pixels showed a response (mean, 12.27 pixels). The degree of activation was greater with a large force (mean, 91.87 pixels). The volume of activation was significantly larger with a large force than with a small force $(p<.01)$.

\subsection{Left hemisphere}

During clenching with a small force, a small number of pixels showed a response (mean, 6.73 pixels). The degree of activation was greater with a large force (mean, 81.28 pixels). The volume of activation was significantly larger with a large force than with a small force $(p<.01)$. Figure 3 shows the activated pixel counts in the right and left hemispheres. There was no significant difference with both large and small forces.

\section{Discussion}

This study investigated the effects of the magnitude of the bite force on neuronal activity in the primary motor cortex. Previous fMRI studies on gum chewing focused on the percentage of changes in fMRI signals, ${ }^{[1-4]}$ although the pixel counts were not measured across slices. The present study showed an increase in the spatial extent of fMRI signals in the primary sensorimotor cortex with an increasing magnitude of bite force.
Table 1: Individual subject values and group data (means and SDs) for the pixel counts with small and large clenching forces

\begin{tabular}{|c|c|c|c|c|}
\hline \multirow{2}{*}{ Subjects } & \multicolumn{2}{|c|}{ Right } & \multicolumn{2}{|c|}{ Left } \\
\hline & Small & Large & Small & Large \\
\hline 1 & 0 & 173 & 0 & 58 \\
\hline 2 & 0 & 24 & 0 & 25 \\
\hline 3 & 14 & 53 & 9 & 81 \\
\hline 4 & 59 & 83 & 28 & 58 \\
\hline 5 & 19 & 37 & 36 & 110 \\
\hline 6 & 2 & 62 & 1 & 24 \\
\hline 7 & 1 & 99 & 1 & 59 \\
\hline 8 & 10 & 44 & 17 & 107 \\
\hline 9 & 26 & 218 & 1 & 121 \\
\hline 10 & 20 & 83 & 5 & 66 \\
\hline 11 & 14 & 131 & 1 & 82 \\
\hline 12 & 7 & 104 & 0 & 99 \\
\hline 13 & 12 & 134 & 2 & 152 \\
\hline 14 & 0 & 74 & 0 & 106 \\
\hline 15 & 0 & 59 & 0 & 71 \\
\hline mean & 12.27 & 91.87 & 6.73 & 81.27 \\
\hline SD & 14.95 & 51.55 & 10.96 & 34.02 \\
\hline
\end{tabular}

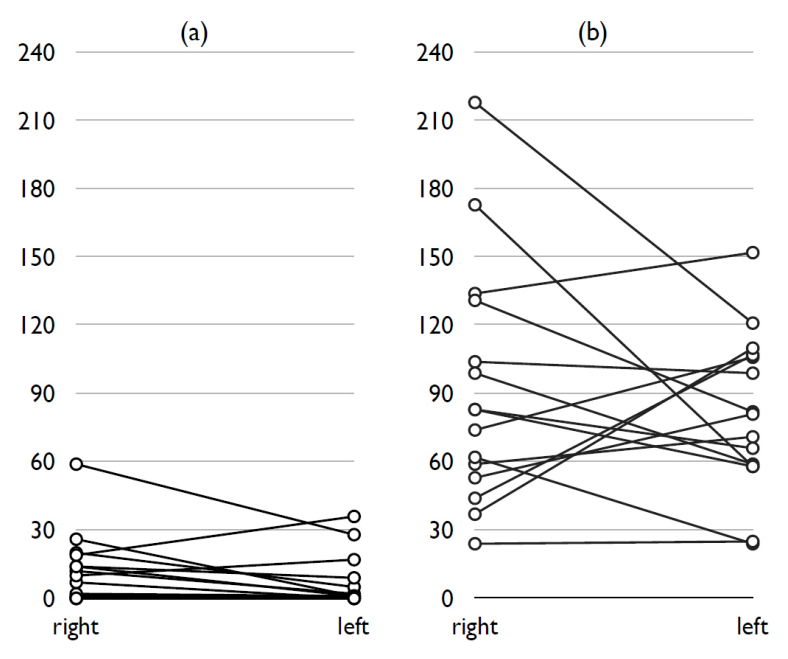

Figure 3: Comparisons of pixel counts with small (a) and large (b) clenching forces between the right and left hemispheres are shown for each of the 15 subjects.

Several factors may explain some of the changes in cortical activation volume with an increasing magnitude of bite force, including an increased neuronal activity ${ }^{[6,14]}$ and the recruitment of larger volumes of the sensorimotor cortex ${ }^{[15]}$ to activate multiple muscle fibers. With a small clenching force, the temporal muscle activity tends to dominate; how- 
ever, both the temporal and masseter muscles show strong activity with a large bite force. ${ }^{[16]}$ Furthermore, increased sensory feedback during clenching with a large force may account for some of the measured changes in cortical activation. It is noteworthy that the increase in the pixel count was remarkable in the region posterior to the central sulcus, probably a sensory area. Increased sensory feedback from the periodontal ligament may remarkably increase cortical activation. The periodontal ligament plays an important role in sensory perception and feedback, which, in turn, tune the motor control.

Previous studies ${ }^{[9-11]}$ that investigated the relationship between force and brain activity involved a squeezing task, which activated only the contralateral sensorimotor cortex. On the other hand, both sensorimotor cortices were activated by clenching because the muscles on both sides contracted at the same time. Therefore, in this study, the activated areas in both sensorimotor cortices were examined and compared. It was hypothesized that nearly equal numbers of pixels were detected in each hemisphere during clenching. However, the intersubject variability was large, and there was a difference between the right and left activated areas. Before fMRI examination, each subject clenched a dental prescale film (Fuji Film) and confirmed that they were exerting a nearly equal and balanced bite force on either side of the dental arches. Therefore, we spec- ulated that a nearly equal bite force may be employed in the early period of the clenching task. However, the force may gradually shift to the habitual side during the 25 -s clenching period.

The major shortcoming of this study was that the exerted bite force or masticatory muscle activity could not be monitored during the fMRI examination. However, our results demonstrate the feasibility of measuring the activated areas in the primary sensorimotor cortex during clenching using fMRI, which revealed that the brain activity is related to the magnitude of the bite force. Therefore, oral rehabilitation with prosthetic treatment, which increases the bite force, ${ }^{[17]}$ not only improves the chewing efficacy but also affects the brain in a positive manner; potentially, the improved bite force may prevent brain degeneration. Additional studies comparing the brain activity before and after prosthetic treatment in edentulous subjects are recommended to evaluate the neurophysiologic effect of prosthetic treatment. Studies examining the measurable bite force and bilateral muscle recruitment during MR scanning can also provide detailed information.

\section{Acknowledgements}

This work was supported by a Grant-in-Aid for Scientific Research (A) (No. 09771561) from the Ministry of Education, Science and Culture, Japan.

\section{References}

[1] Tamura T, Kanayama T, Yoshida S, Kawasaki T. Analysis of brain activity during clenching by fMRI. J Oral Rehabil. 2002; 29: 467-472. PMid: 12028495. http://dx.doi.org/10.1046/j.1 365-2842.2002.00880.x

[2] Onozuka M, Fujita M, Watanabe K, Hirano Y, Niwa M, Nishiyama $\mathrm{K}$, et al. Mapping brain region activity during chewing: a functional magnetic resonance imaging study. J Dent Res. 2002; 81: 743-746. PMid: 12407087. http://dx.doi.org/10.1177/15440591020 8101104

[3] Tamura T, Kanayama T, Yoshida S, Kawasaki T. Functional magnetic resonance imaging of human jaw movements. J Oral Rehabil. 2003; 30: 614-622. PMid: 12787459. http://dx.doi.org/10. 1046/j.1365-2842.2003.01054.x

[4] Onozuka M, Fujita M, Watanabe K, Hirano Y, Niwa M, Nishiyama $\mathrm{K}$, et al. Age-related changes in brain regional activity during chewing: a functional magnetic resonance imaging study. J Dent Res. 2003; 82: 657-660. PMid: 12885854. http://dx.doi.org/10. $1177 / 154405910308200817$

[5] Evarts EV. Relation of pyramidal tract activity to force exerted during voluntary movement. J Neurophysiol. 1968; 43: 1070-1089.

[6] Cheney PD, Fetz EE. Functional classes of primate corticomotoneuronal cells and their relation to active force. J Neurophysiol. 1980; 44: 773-791. PMid: 6253605.

[7] Hepp-reymond MC, Wyuss UR, Anner R. Neuronal coding of static force in the primate motor cortex. J Physiol. 1978; 74: 287-291.

[8] Georgopoulos AP, Ashe J, Smyrnis N, Taira M. The motor cortex and the coding of force. Science. 1992; 256: 1692-5. PMid:
1609282. http://dx.doi.org/10.1126/science.256.5064. 1692

[9] Detters C. Fink GR, Lemon RN, Stephan KM, Passingham RE, Silbersweig $\mathrm{D}$, et al. Relation between cerebral activity and force in the motor areas of the human brain. J Neurophys. 1995; 74: 802-815.

[10] Ehrsson HH, Fagergren A, Jonsson T, Westling G, Johansson RS, Forssberg H. Cortical activity in precision- vs. power-grip tasks: an fMRI study. J Neurophysiol. 2000; 83: 528-536. PMid: 10634893.

[11] Thickbroom GH, Phillips BA, Morris I, Bymes MI, Mastaglia FL. Isometric force-related activity in sensorimotor cortex measured with functional MRI. Exp Brain Res. 1998; 121: 59-64. PMid: 9698191. http://dx.doi.org/10.1007/s002210050437

[12] Ludman CN, Cooper TG, Ploutz-synder LL, Potchen EJ, Meyer RA. Force of voluntary exercise does not affect sensorimotor cortex activation as detected by functional MRI at $1.5 \mathrm{~T}$. NMR Biomed. 1996; 9: 228-232. http://dx.doi.org/10.1002/(SICI) 109 9-1492(199608) 9:5<228: :AID-NBM420>3.0.CO;2-1

[13] Wexler, BE, Fulbright, RK, Lacadie, CM, Skudlarski, P, Kelz, MB, Constable, RT, et al. An fMRI study of the human cortical motor system response to increasing functional demands. Magn Reson Imaging. 1997; 15: 385-96. http://dx.doi.org/10.1016/S0730-7 25X (96) 00232-9

[14] Wannier TMJ, Maier MA, Hepp-reymond M-C. Contrasting properties of monkey somatosensory and motor cortex neurons activated during the control of force and precision grip. J Neurophysiol. 1991; 65: 572-589. PMid: 2051196.

[15] Steven C Cramer, Robert M Weisskoff, Judith D Schaechter, Gereon Nelles, Mary Foley, Seth P Finklestein, et al. Motor cortex activation is related to force of sqsueezing. Human Brain Mapping. 2002; 
16: 197-205. PMid: 12112762. http://dx.doi.org/10.1002/h bm. 10040

[16] Naeije, M., McCarroll, R. S., Weijs, W. A. Electromyographic activity of the human masticatory muscles during submaximal clenching in the inter-cuspal position. J Oral Rehabil. 1989; 16: 63-70. PMid: 2746406. http://dx.doi.org/10.1111/j.1365-2842. 1989.tb01318.x

[17] Fontijn-Tekamp, FA, Slagter, AP, van't Hof, MA, Geertman, ME, Kalk, W. Bite forces with mandibular implant-retained overdentures. J Dent Res. 1998; 77: 1832-9. PMid: 9786640. http://dx. doi.o $\mathrm{rg} / 10.1177 / 00220345980770101101$ 